\title{
Advanced Imaging of the Vestibular Endolymphatic Space in Ménière's Disease
}

\author{
Diego Zanetti ${ }^{1,2 *}$, Giorgio Conte ${ }^{3 \dagger}$, Elisa Scola ${ }^{3 \dagger}$, Silvia Casale ${ }^{3 \dagger}$, Giorgio Lilli $^{1,2 \dagger}$ and \\ Federica Di Berardino ${ }^{1,2+}$
}

OPEN ACCESS

Edited by:

Robert Gürkov

Bielefeld University, Germany

Reviewed by:

Sebastian Christoph Roesch,

Paracelsus Medical University, Austria

Hans Thomeer,

University Medical Center

Utrecht, Netherlands

*Correspondence:

Diego Zanetti

diego.zanetti.bs@gmail.com

TORCID:

Diego Zanetti

orcid.org/0000-0002-8116-4108

Giorgio Conte

orcid.org/0000-0002-1562-7389

Elisa Scola

orcid.org/0000-0001-9056-4122

Silvia Casale

orcid.org/0000-0002-6286-7537

Giorgio Lilli

orcid.org/0000-0002-6109-5037

Federica Di Berardino

orcid.org/0000-0001-7514-5624

Specialty section:

This article was submitted to

Otorhinolaryngology-Head and Neck

Surgery,

a section of the journal

Frontiers in Surgery

Received: 25 April 2021

Accepted: 19 July 2021

Published: 23 August 2021

Citation:

Zanetti D, Conte G, Scola E, Casale S,

Lilli $G$ and Di Berardino F (2021)

Advanced Imaging of the Vestibular

Endolymphatic Space in Ménière's

Disease. Front. Surg. 8:700271.

doi: 10.3389/fsurg.2021.700271
'Audiology Unit, Department of Specialistic Surgical Sciences, Fondazione IRCCS Ca' Granda Ospedale Maggiore Policlinico, University of Milan, Milan, Italy, ${ }^{2}$ Audiology Unit, Department of Clinical Sciences and Community Health, Fondazione IRCCS Ca' Granda Ospedale Maggiore Policlinico, University of Milan, Milan, Italy, ${ }^{3}$ Neuroradiology Unit, Fondazione IRCCS Ca' Granda Ospedale Maggiore Policlinico, Milan, Italy

The diagnosis of "definite" Méniére's disease (MD) relies upon its clinical manifestations. MD has been related with Endolymphatic Hydrops $(E H)$, an enlargement of the endolymphatic spaces (ES) (cochlear duct, posterior labyrinth, or both). Recent advances in Magnetic Resonance (MR) imaging justify its increasing role in the diagnostic workup: $\mathrm{EH}$ can be consistently recognized in living human subjects by means of 3-dimensional Fluid-Attenuated Inversion-Recovery sequences (3D-FLAIR) acquired $4 \mathrm{~h}$ post-injection of intra-venous (i.v.) Gadolinium-based contrast medium, or $24 \mathrm{~h}$ after an intratympanic (i.t.) injection. Different criteria to assess $\mathrm{EH}$ include: the comparison of the area of the vestibular ES with the whole vestibule on an axial section; the saccule-to-utricle ratio ("SURI"); and the bulging of the vestibular organs toward the inferior $1 / 3$ of the vestibule, in contact with the stapedial platina ("VESCO"). An absolute link between MD and EH has been questioned, since not all patients with hydrops manifest MD symptoms. In this literature review, we report the technical refinements of the imaging methods proposed with either i.t. or i.v. delivery routes, and we browse the outcomes of MR imaging of the ES in both $\mathrm{MD}$ and non-MD patients. Finally, we summarize the following imaging findings observed by different researchers: blood-labyrinthine-barrier (BLB) breakdown, the extent and grading of $\mathrm{EH}$, its correlation with clinical symptoms, otoneurological tests, and stage and progression of the disease.

Keywords: magnetic resonance imaging, hydrops, membranous labyrinth, ear, vestibule

\section{INTRODUCTION}

Ménière's disease (MD) is thought to be an unbalance of inner ear fluids, manifesting as fluctuating sensorineural hearing loss (SNHL), recurrent vertigo spells, tinnitus, and ear fullness (1). It affects between 200 and 500 persons every 100,000 inhabitants in Western countries (2). Endolymphatic Hydrops (EH), a swelling of the endolymphatic space (ES) that occupies part of the perilymphatic space (PS) constitutes the pathological landmark of the disease (3). It encompasses the cochlear duct and the saccule, sometimes extending to the semicircular canals (SCC) and utricle.

In 1995, the American Academy of Otolaryngology-Head and Neck Surgery (AAO-HNS) reestablished the guidelines for the assessment of MD (4). In 2015 (5), a new consensus was reached on 2 main forms of MD "definite" and "probable" MD. Diagnosis of definite MD is obtained upon two or more vertigo spells limited to a period between $20 \mathrm{~min}$ and $12 \mathrm{~h}$, associated with unilateral 
low-frequencies SNHL, tinnitus and intermittent ear fullness. Probable MD is defined by more than 2 vertigo spells lasting between 20 min and 1 day and ipsilateral intermittent ear fullness. To evaluate the presence of EH, electrocochleography (ECochG) is able to identify, through an acoustic stimulation, the increase of the endolymphatic pressure with bulging of the Reissner's membrane in case of EH. Other electrophysiological tests, such as the vestibular evoked myogenic potentials (VEMPs) and video head impulse test (vHIT) might be useful, but their role is still controversial.

Recent reports confirm that the otoneurological responses correlate well with the magnetic resonance imaging (MRI) showing that the $\mathrm{EH}$ starts from the saccule and then progresses to the SCC (6).

In the past, imaging studies were still obtained to exclude retrocochlear disorders, such as vestibular nerve schwannomas. New developments of MRI techniques (7) have enabled visualization of $\mathrm{EH}$ in humans by means of 3 Tesla (3T) scanners and Gadolinium (Gd) administered either intravenously (i.v.) (8) or intratympanically (i.t.) $(9,10)$. However, the diagnostic accuracy of $\mathrm{EH}$ by MRI is disputed, mainly owing to the disparities in the inclusion and diagnostic criteria.

The clinical consequences of a correct assessment of $\mathrm{EH}$ would represent a significant leap forward, by providing a more reliable tool to differentiate between $\mathrm{MD}$ and non-MD diseases of the inner ear $(11,12)$ to follow-up the untreated clinical evolution of the disease (13) or to test different pharmacological or surgical protocols $(14,15)$. The purpose of this review of the literature is to understand the reliability of MRI in detecting $\mathrm{EH}$ in the population of suspected MD patients with insights of the contemporary technological advances and to compare the imaging findings with the natural history of the disease and the outcome of treatments. Furthermore, we discuss the newest $\mathrm{MR}$ protocols to assess $\mathrm{EH}$ and the commonest MRI findings in different vestibular disorders.

\section{Literature Search Strategy}

According to the PRISMA guidelines (16), the scientific literature of the last decade was browsed with the aim of identifying studies describing the MR findings in "definite" MD patients (see the PRISMA flowchart in the additional digital content).

The following search queries were used in Medline, Cochrane database, Scopus and EMBASE (from 2010 to Present): "Ménière" OR "endolymphatic hydrops" AND "magnetic resonance" OR "MR." The search was restricted to articles that provided at least an abstract in English. References of the selected publications were also examined to extract any further relevant article. The retrieved articles were considered eligible if they provided results of MR in patients with "definite" MD (4) or to the 2015 Consensus Statement (5). Two of the co-Authors (FDB and GL) separately reviewed all articles and excluded those with unclear clinical diagnostic criteria. Other exclusion criteria were studies on animals, case reports, personal (expert) opinions, metanalysis, different diagnostic criteria, studies where diagnosis of "definite" MD was lacking; studies not assessing hydrops by accepted MRI techniques; and studies not reporting the status of the contralateral (unaffected) ear.
The following information were outsourced from the selected studies: first author, year of publication, total number of subjects enrolled, MRI techniques and peculiar MRI findings related with the presence of hydrops in the affected vs. unaffected ears.

\section{Literature Search Results}

The initial search yielded a total of 219 articles: 97 articles in PubMed, 91 in EMBASE, 27 in Scopus and none in the Cochrane Library. After excluding duplicates and those not respecting the inclusion criteria, 77 articles were left; among these, 47 were included by relevance. These 47 papers were carefully analyzed for the purposes of this study. Six of them were review studies and were only considered for the general overview of the topic.

The main features of each of the 41 remaining articles are reported in Table 1: 8 out of 41 studies performed an i.t. administration of Gd, 27 only an i.v. delivery and 3 studies performed both simultaneously; 3 studies dealt with postacquisition processing of images.

The MR images were acquired $4 \mathrm{~h}$ after the i.v administration in 26 out of 27 studies, and $24 \mathrm{~h}$ after the i.t. injection in 8 out of 11 trials (8 i.t. only and 3 i.t. + i.v).

A 3T machine was used in 40 out of 41 studies. The most common MRI technique to identify EH was the $3 \mathrm{D}$ FLAIR ( $n=$ $38 / 41$ original studies).

The selected method to assess the degree of $\mathrm{EH}$ was the VES/vestibule ratio $>30 \%$ in 28 studies, $>50 \%$ in 3 studies, the SURI in 4 studies, the VESCO only in 1 study.

The sensitivity, specificity, PPV and NPV of the techniques in correctly identifying EH were reported in 37 out of 41 studies.

Side effects of Gd administration were reported in 3 of 1,578 patients (sum of all articles), all of them with the i.t. routes.

\section{DISCUSSION}

The published papers on MRI of the inner ear have almost doubled during the last 10 years, confirming the interest in the subject and the potentiality of the innovative methods. However, the role of MRI in differentiating between different inner ear diseases remains to be established. Based on general opinion in the field, we considered reasonable to report on the volumetric detection of $\mathrm{EH}$ rather than generic imaging of MD.

The MRI identification of the fluid compartments of the inner ear can be achieved by either intratympanic or intravenous administration of Gd (8).

\section{The Intra-Tympanic Gadolinium Injection}

It has been the first method proposed in the literature to study the inner ear spaces $(9,54)$. The i.t.-Gd delivery method utilizes an i.t. injection of $0.4-0.7 \mathrm{ml}$ of an 8 -fold dilution of the Gd solution into the tympanic cavity. The contrast medium diffuses into the perilymph through the round window membrane (RWM), but not in the endolymph, producing the socalled perilymph positive image (PPI). Considering the diffusion dynamics, the MRI scans are obtained 1 day after the i.t. injection. The method of choice is based on T2-weighted scans with the 3D Fluid-Attenuated-Inversion-Recovery (3D-FLAIR) 
TABLE 1 | Summary of literature review (last decade) on MRI in Meniere's disease (MD).

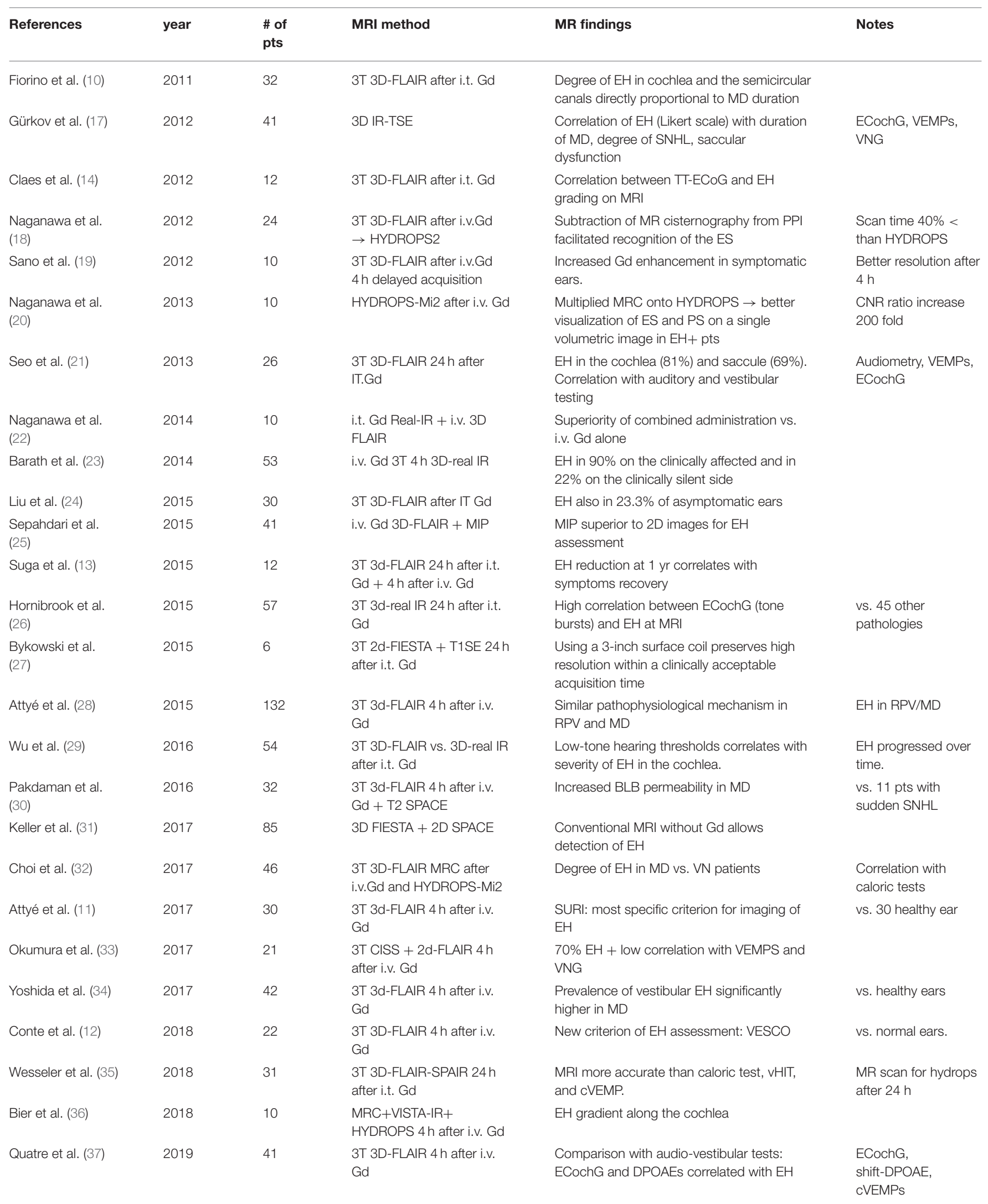


TABLE 1 | Continued

\begin{tabular}{|c|c|c|c|c|c|}
\hline References & year & $\begin{array}{l}\text { \# of } \\
\text { pts }\end{array}$ & MRI method & MR findings & Notes \\
\hline $\begin{array}{l}\text { Pérez-Fernández } \\
\text { et al. (38) }\end{array}$ & 2019 & 22 & $\begin{array}{l}\text { 3T 3D-FLAIR } 4 \mathrm{~h} \text { after i.v. } \\
\text { Gd }\end{array}$ & $\begin{array}{l}\text { Degree of vEH correlates with vestibular } \\
\text { deficit }\end{array}$ & $\begin{array}{l}\text { vHIT, VEMPs, } \\
\text { audiometry }\end{array}$ \\
\hline $\begin{array}{l}\text { Bernaerts et al. } \\
\text { (39) }\end{array}$ & 2019 & 148 & $\begin{array}{l}\text { 3T 3D-FLAIR } 4 \text { h after i.v. } \\
\text { Gd }\end{array}$ & $\begin{array}{l}4 \text { degrees of EH. Combined with } \\
\text { perilymphatic enhancement: Sensitivity } \\
79.5 \% \text { Specificity } 93.6 \%\end{array}$ & \\
\hline Ito et al. (15) & 2019 & 20 & $\begin{array}{l}\text { 3T 3D-FLAIR + MRC } 4 \mathrm{~h} \\
\text { after i.v. Gd }\end{array}$ & $\begin{array}{l}\text { Reduction of EH } 2 \text { ys after endolymphatic } \\
\text { sac drainage and local steroids }\end{array}$ & $\begin{array}{l}\text { pre- and } \\
\text { post-surgery MR }\end{array}$ \\
\hline Eliezer et al. (40) & 2019 & 30 & $\begin{array}{l}\text { 3T 3D-FLAIR + SSFP } 4 \mathrm{~h} \\
\text { after i.v. Gd }\end{array}$ & In acute vestibular deficits & vs. healthy ears \\
\hline Guo et al. (41) & 2019 & 56 & $\begin{array}{l}\text { 3T T2-SPACE and Real-IR } \\
4 \text { h after i.v. Gd }\end{array}$ & $\begin{array}{l}\text { Degree of EH correlates with audiometry } \\
\text { and VEMPs }\end{array}$ & $\begin{array}{l}\text { oVEMP and PTA } \\
\text { correlates with } \\
\text { cochlear EH }\end{array}$ \\
\hline Ohashi et al. (42) & 2020 & 15 & $\begin{array}{l}\text { HYDROPS-Mi2 + 3D-real } \\
\text { IR images }\end{array}$ & Measurement of endolymphatic volume & $\begin{array}{l}\text { Comparison of } 2 \\
\text { techniques }\end{array}$ \\
\hline Gerb et al. (43) & 2020 & 105 & $\begin{array}{l}\text { 3T 3D-FLAIR + CISS } 4 \mathrm{~h} \\
\text { after i.t. Gd }\end{array}$ & $\begin{array}{l}\text { VOLT: new algorithm for automatic } \\
\text { segmentation of MR images }\end{array}$ & $\begin{array}{l}\text { All pts with acute } \\
\text { vertigo (incl. MD) }\end{array}$ \\
\hline Nahmani et al. (44) & 2020 & 16 & $\begin{array}{l}\text { 3T 3D-FLAIR } 4 \mathrm{~h} \text { after i.v. } \\
\text { Gd Variable Flip Angle- }\end{array}$ & $\begin{array}{l}\text { Reliable in evaluating BLB breakdown and } \\
\text { ES }\end{array}$ & \\
\hline Cho et al. (45) & 2020 & 226 & $\begin{array}{l}\text { INHEARIT on archived } \\
\text { datasets }\end{array}$ & $\begin{array}{l}\text { Feasibility of automated EH ratio } \\
\text { measurements }\end{array}$ & $\begin{array}{l}\text { Automatic } \\
\text { segmentation }+ \\
\text { calculation of } \mathrm{EH}\end{array}$ \\
\hline $\begin{array}{l}\text { van Steekelenburg } \\
\text { (46) }\end{array}$ & 2020 & 220 & $\begin{array}{l}\text { 3T 3D-FLAIR + SPACE } 4 \mathrm{~h} \\
\text { after i.v. Gd }\end{array}$ & $\mathrm{EH}$ in $91.9 \%$ of $\mathrm{MD}$ vs. $7 \%$ in other vertigo. & $\begin{array}{l}\text { Combination of PPI } \\
\text { and } \mathrm{EH} \text { in } \mathrm{MD}\end{array}$ \\
\hline Pai et al. (47) & 2020 & 31 & $\begin{array}{l}\text { 3T 3D-FLAIR } 4 \mathrm{~h} \text { after i.v. } \\
\text { Gd }\end{array}$ & $\begin{array}{l}\text { Correlation of } \mathrm{EH} \text { finding at MR with } \\
\text { diseased ear }\end{array}$ & \\
\hline $\begin{array}{l}\text { Fukushima et al. } \\
\text { (48) }\end{array}$ & 2020 & 55 & $\begin{array}{l}\text { 3T 3D-FLAIR and } \\
\text { HYDROPS-Mi2 } 4 \mathrm{~h} \text { after i.v. } \\
\text { Gd }\end{array}$ & Progression of vestibular EH over 2-3 years & MR repeated annually \\
\hline Kahn et al. (49) & 2020 & 31 & $\begin{array}{l}\text { 3T 3D-FLAIR } 4 \mathrm{~h} \text { after i.v. } \\
\text { Gd }\end{array}$ & $\begin{array}{l}\text { EH: Cochlear } 88 \% \text {, saccular } 91 \% \text {, utricular } \\
50 \% \text {, ampullar } 8.5 \% \text {. No correlation with } \\
\text { VEMPs/vHIT. Severity of EH correlated with } \\
\text { SNHL }\end{array}$ & vs. 26 healthy ears \\
\hline Gürkov et al. (50) & 2021 & 30 & $\begin{array}{l}\text { 1.5T 3D-FLAIR Hydrops } \\
24 \text { h after i.t. Gd }\end{array}$ & $\begin{array}{l}\text { Reliability of } 1.5 \mathrm{~T} \text { fast identification and } \\
\text { grading of } \mathrm{EH}\end{array}$ & \\
\hline He et al. (51) & 2021 & 50 & $\begin{array}{l}\text { 3T 3D-FLAIR } 4 \mathrm{~h} \text { after i.v. } \\
\text { Gd }\end{array}$ & $\begin{array}{l}\text { 3D-FLAIR MRI + PT- ECochG more } \\
\text { sensitive than ECochG alone for EH }\end{array}$ & PT- ECochG \\
\hline Zhang et al. (52) & 2021 & 24 & $\begin{array}{l}\text { 3T 3D-FLAIR } 4 \mathrm{~h} \text { after i.v. } \\
\text { Gd }\end{array}$ & $\begin{array}{l}\text { Degree of EH correlates with hearing } \\
\text { threshold }\end{array}$ & Extratympanic \\
\hline Sluydts et al. (53) & 2021 & 78 & $\begin{array}{l}\text { 3T 3D-FLAIR } 4 \mathrm{~h} \text { after i.v. } \\
\text { Gd }\end{array}$ & $\begin{array}{l}\text { Only severe cochlear and vestibular } \mathrm{EH} \text { are } \\
\text { associated with cochleovestibular } \\
\text { dysfunction }\end{array}$ & $\begin{array}{l}\text { Audiometry, caloric } \\
\text { tests, VEMPs, vHIT }\end{array}$ \\
\hline
\end{tabular}

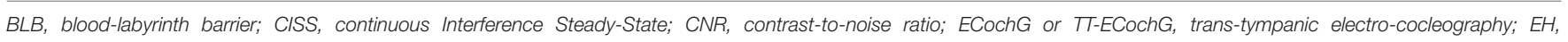

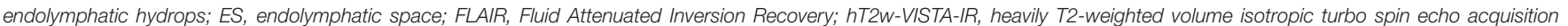

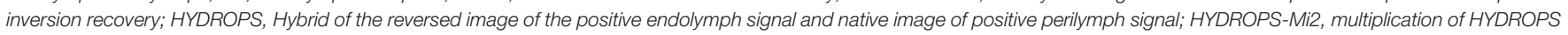

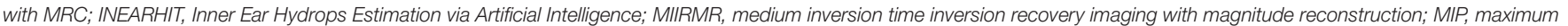

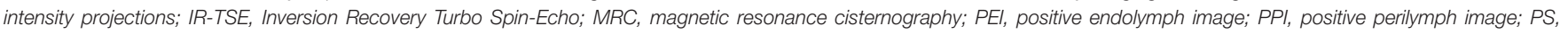

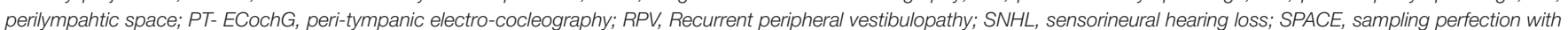

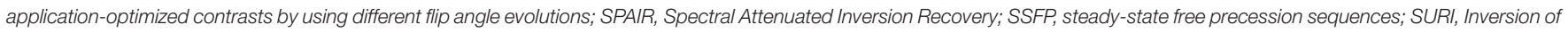

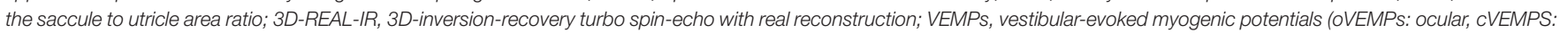
cervical); VESCO, vestibular endolymphatic space contacting the oval window; VHIT, video Head Impulse Test; VNG, video-nystagmography; VN, vestibular neuritis.

algorithm, which lowers the endolymph signal in respect to the adjacent perilymph. Variations to the flip angle can be applied. If the inversion time of the 3D-FLAIR is shortened, the perilymph signal is suppressed and that of the endolymph is enhanced, obtaining a positive endolymphatic image (PEI).
Another technique, namely 3D-inversion-recovery turbo spinecho (TSE) with real reconstruction (3D-real IR) (55) creates a sharp contrast between the inner ear fluids (the positive perilymph vs. the negative endolymph) and the neighboring bone that results as null. 
Given the known entry routes and kinetics of drugs from the middle to the inner ear, the most suitable and reliable method to investigate the vestibular $\mathrm{EH}(\mathrm{vEH})$ and the cochlear $\mathrm{EH}(\mathrm{cEH})$ is that described by Nakashima et al. (9). In contrast, Carfrae et al. (54) and Shi et al. (55) denied an additional value of $\mathrm{Gd}$ delivered directly to the middle ear during surgery: $\mathrm{vEH}$ and $\mathrm{cEH}$ were detected only in 25 and $16 \%$ of patients, respectively, possibly owing to the dilution of the contrast medium. In many countries, the i.t. delivery of Gd is still off-label; moreover, some patients are reluctant to undergo bilateral injections of their tympanic membranes. Thus, the puncture is limited to the affected ear, leaving the contralateral uninvestigated.

$\mathrm{Wu}$ et al. (29) administered a bilateral i.t. injection of Gd: the presence of $\mathrm{vEH}$ was detected in two thirds of the ears with clinical symptoms of MD, while cEH in $8 \%$. A few studies tested the simultaneous delivery of Gd through the i.t. and the i.v. routes: Iida et al. (56) demonstrated the presence of both $\mathrm{vEH}$ and $\mathrm{cEH}$ in $67 \%$ of the contralateral asymptomatic ears. Naganawa et al. (22) used 3D-real IR images for the i.t.-Gd side and the so-called HYDROPS sequences ("Hybrid of the reversed image of the positive endolymph signal and native image of positive perilymph signal") for i.v perfusion. Only HYDROPS images were able to demonstrate vEH in $89 \%$ and $\mathrm{cEH}$ in $67 \%$ of all symptomatic ears, respectively. The i.t. administration is able to show the presence of $\mathrm{EH}$ in other audiological disorders $(26,57)$, even if at a very low rate.

To improve the acquisition and analysis of the images, Bykowski et al. (27) applied 8-channel surface coils to acquire 3D-FLAIR images after an i.t. injections in six patients with definite MD. By varying the inversion times, they were able to judge the fluid-suppression ability of each sequence, in all the six patients tested.

\section{The Intra-Venous Gadolinium Perfusion}

Although the i.t.- Gd technique has the great benefit of enhancing the visualization of the perilymph (35), the i.v.-Gd delivery route has several advantages: lesser invasiveness (although major complications can still occur); reduced operating times $(4 \mathrm{~h}$ for a comprehensive MRI study); bilateral examination in a single test session. It consists in the intravenous perfusion of a fixed (per weight) dose of Gd (between 0.1 and 0.2 $\mathrm{ml} / \mathrm{Kg}$ ) that rather quickly diffuses in the perilymph without spreading to the endolymph, depending on the permeability of the blood-labyrinth barrier (BLB) (58), thus creating a PPI. The 2 most popular scanning sequences, 3D-real IR and 3D-FLAIR are substantially equivalent, although the latter is more sensitive to less concentrated dilutions of Gd (12).

Noteworthy, a correct visualization of the inner ear compartments relies upon the inversion time. After the normal MR standards are established in healthy controls, MR images can be immediately analyzed after acquisition or they can be scrutinized after processing. Post-processing includes subtraction of the PEI (a 3D FLAIR sequence) from the PPI. HYDROPS and HYDROPS2 (22) are then reconstructed and can be juxtaposed to the corresponding MR cisternography images to obtain the HYDROPS-Mi2 and HYDROPS2-Mi2 images, respectively, which show increased contrast compared to background noise (42). The combination of "maximum intensity projection" (MIP) and 3D-FLAIR further adds robustness to the assessment of $\mathrm{EH}$.

The main concern about either technique of MRI of $\mathrm{EH}$ is the correct identification of the vestibular organs, which requires a thorough knowledge of the radiological anatomy. During the last decade, a number of different methods to detect hydrops have been proposed, raising also a vivid debate among research groups.

Nakashima et al. (59) initially described the vestibular endolymphatic space (VES) by calculating the ratio between the endolymphatic organs and the whole vestibule in an axial projection. They defined the $\mathrm{vEH}$ as "absent" when the ratio was $<33 \%$, "mild" when $34-50 \%$ and "significant" if $>50 \%$. In addition, they evaluated the cochlear ES (cES) by measuring the displacement of the Reissner's membrane. They defined the cEH as "mild" if the displacement did not exceed the scala vestibuli (SV), or "significant" when the cES exceeded the SV.

In clinical studies, the first problem encountered with the $\mathrm{VES} /$ vestibule ratio was that mild $\mathrm{vEH}$ was not only reported in the greatest majority of symptomatic ears of MD patients, but also in more than half of the asymptomatic contralateral ears $(19,34,58,60)$. Moreover, $\mathrm{EH}$ has been detected also in healthy individuals $(11,61,62)$, and this challenges its correlation with MD (63).

A few studies also raise the issue of specificity: mild vEH was identified even in symptomatic ears of patients with otological diseases other than MD (19), such as recurrent peripheral vestibulopathy and unilateral labyrinthine deficit $(28,40)$.

Nevertheless, the sensitivity of i.v. Gd-enhanced MR with $4 \mathrm{~h}$ delayed acquisitions is very high ( $>95 \%$ ); thus, a significant VES/vestibule ratio might represent a potential indicator of MD even before a clear clinical diagnosis has been established. In fact, severe vEH was always excluded the ears of clinically silent MD patients (30) and mild vEH was in the very low range (below 20\%) in healthy volunteers. Considering a VES/vestibule ratio $>50 \%$ (instead of $30 \%$ ) may reduce its sensitivity but can represent a more reliable rule-in criteria for $\mathrm{MD}$, allowing to differentiate $\mathrm{MD}$ from unaffected ears and from other auricular diseases.

Although Nakashima's criteria are universally accepted, other cut-off values for vEH have been suggested: Sepahdari et al. (25) used a VES/vestibule ratio of 45\% (2 SD above the mean) in a group of patients with sudden SNHL and found $\mathrm{vEH}$ in $6 / 12(50 \%)$, concluding that it was inconsistent for the assessment of $\mathrm{MD}$ in sudden SNHL. Another study (34) claimed that even a lower ratio of $41.9 \%$ yielded an absolute specificity of $100 \%$ in differentiating hydropic from normal ears, still retaining a high sensitivity (88.5\%). They also studied the asymptomatic ears and 

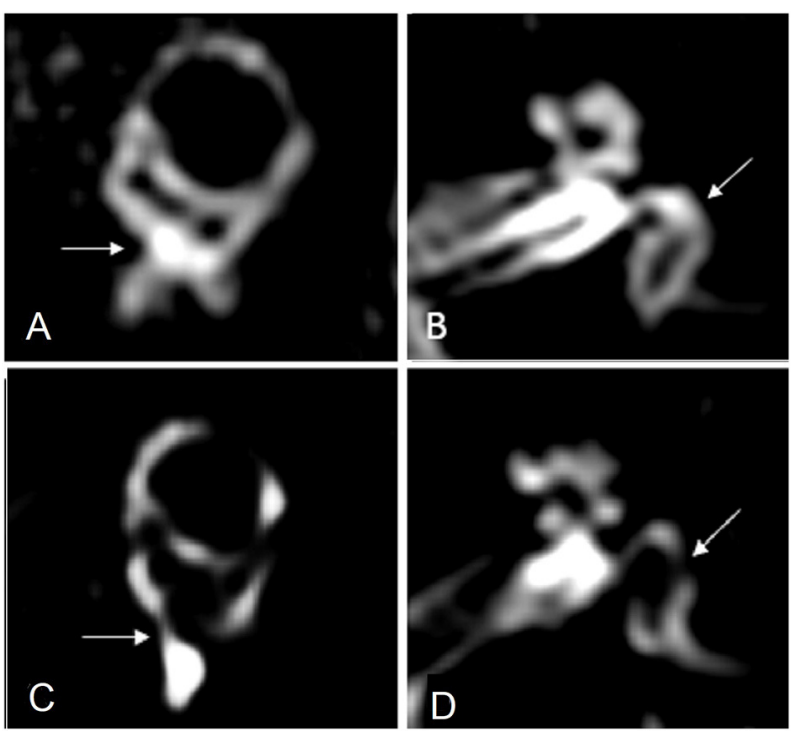

FIGURE 1 | (A) MR imaging oblique sagittal reconstruction parallel to the superior semicircular canal of a healthy ear shows superiorly the vestibular endolymphatic space and inferiorly the perilymph filling the inferior third of the vestibule with preservation of the perilymph signal medial to the oval window (arrow). (B) MR imaging axial reconstruction parallel to the lateral semicircular canal at the inferior third of the vestibule in a healthy subject, showing the vestibule filled by the perilymph (arrow). (C) MR imaging oblique sagittal reconstruction parallel to the superior semicircular canal in a patient with Menière disease shows enlargement of the vestibular endolymphatic space bulging into the inferior third of the vestibule and contacting the oval window (arrow), with the consequent absence of the normal perilymph signal. (D) MR imaging axial reconstruction parallel to the lateral semicircular canal at the inferior third of the vestibule shows the vestibular endolymphatic space contacting the oval window, "VESCO" (arrow).

ascertained $\mathrm{cEH}$ in $46 \%$ of $\mathrm{MD}$ patients and $33 \%$ of healthy subjects.

Other research groups graded the $\mathrm{EH}$ with a criterium based on the morphology of the saccule $(11,28,40)$. Using a ratio between the area of the saccule and that of the utricle (SURI) $>50 \%$, they were able to differentiate the ears of 30 patients with MD from normal controls, with a sensitivity of $50 \%$ and specificity of 100 . The reliability of post-contrast imaging in detecting EH was very high in Eliezer et al. study (64) by means of $3 \mathrm{D}$-FLAIR and $4 \mathrm{~h}$ delayed acquisition, with much greater efficiency than the 3D FIESTA sequences.

Conte et al. (12) compared the imaging outcomes in 49 subjects, half of which had suffered a sudden SNHL, and the remaining were afflicted by definite $\mathrm{MD}$. Using the 4-h delayed 3D-FLAIR protocol, 2 independent examiners observed that in MD patients the saccule was swollen and protruded in the lower part of the vestibule, arriving in contact with the footplate of the stapes. They named this finding "VESCO" an acronym of "vestibular endolymphatic space contacting the oval window." The VESCO showed an optimal specificity but a low sensitivity (81\%) in differentiating MD ears from other inner ear diseases. Figure 1 details the regular membranous labyrinth anatomy in a $3 \mathrm{~T} \mathrm{Gd-}$ FLAIR axial scan in one of our healthy adult volunteers and depicts the VESCO in one of our symptomatic MD patients.

\section{Recent Advances}

A number of technical innovations have been recently added to the MR armamentarium to improve the identification of $\mathrm{EH}$, such as the Variable and Constant Flip Angle-Delayed 3D-FLAIR Sequences (44) or the i.t.-Gd "medium inversion time inversion recovery imaging with magnitude reconstruction" (MIIRMR) (65).

In order to eliminate the subjective (examiner's) bias, new automated images segmentation processing algorithms have been introduced $(43,66)$, also associated with deep-learning models based on Artificial Intelligence (45). By adding the quantification of perilymphatic enhancement to the grading of $\mathrm{EH}$, van Steekelenburg et al. (46) recently reported to improve the positive predictive value of Gd-enhanced MRI from 0.92 to 0.97 in the confirmation of definite MD.

The majority of ongoing studies is currently aimed at targeting the correlation between the morphologic findings and the symptomatology ("the whole symptoms triad") and, especially, with the results of the functional audio-vestibular testing and with the outcomes of treatment $(37,41,48,51,52,62,63,67)$. In general, the literature agrees that the presence of $\mathrm{EH}$ at MRI, independently from the cut-off values for definition of $\mathrm{vEH}$, strongly correlates with the side of the disease in MD patients $(47,50)$, but it lacks specificity in differentiating MD from other inner ear disturbances, in the absence of clinical/instrumental confirmation (64). It seems that the MRI demonstration of $\mathrm{EH}$ is a necessary but not sufficient condition to assess a diagnosis of MD. Nevertheless, it is highly improbable that $\mathrm{EH}$ is only casually associated with $\mathrm{MD}$, because the causative relationship is too stringent (2).

The present review has some limitations: the reported populations are rather heterogenous in diagnostic criteria and definition of $\mathrm{MD}$; some studies include very small patients' samples, thus a metanalysis was not feasible. In contrast, the review offers an insight of the latest technological developments.

In conclusion, current MR imaging methods allow to clearly depict the ES vs. the PS, in both the cochlear and vestibular compartments with multiple dedicated protocols. MRI of the ES can be comfortably obtained by means of i.v. administration of $\mathrm{Gd}$ and late $(4 \mathrm{~h})$ acquisitions. Thus, the more invasive and offlabel i.t. injection of Gd, is considered by most Authors a 2nd-line investigative tool.

Although the role of $\mathrm{EH}$ in $\mathrm{MD}$ has been questioned by recent research, and its presence may not always be considered pathological, current MR imaging yields a very high sensitivity in detecting it. As quantitative indexes alone are probably insufficient to establish an MRI diagnosis of MD, more accurate criteria based on the morphology of the endolymphatic organs are required. A 3 Tesla MR scanner is essential for the purpose of identifying the subtle morphological variations of the ES in MD and to correlate the findings with the clinical history and audio-vestibular testing. The newest techniques proposed in the last years appear to be promising tools and deserve to be further investigated, especially focusing on the features, prevalence and role of $\mathrm{EH}$ in different inner ear disorders and on the relevance of other findings (BLB breakdown, methemoglobin, and inflammatory deposits) in the ES. 


\section{AUTHOR CONTRIBUTIONS}

GC defined the literature search strategy, evaluated the articles relevance, and revised the manuscript. ES scrutinized the retrieved articles for inclusion/exclusion in the systematic review. SC scrutinized the retrieved articles for inclusion/exclusion in the systematic review and provided the MR images with captions. GL prepared the draft of the manuscript. FDB revised the manuscript and checked references and table. $\mathrm{DZ}$ designed the study, revised, and edited the final version of the manuscript. All authors contributed to the article and approved the submitted version.

\section{REFERENCES}

1. Basura GJ, Adams ME, Monfared A, Schwartz SR, Antonelli PJ, Burkard R, et al. Clinical practice guideline: Ménière's disease. Otolaryngol Head Neck Surg. (2020) 162:S1-55. doi: 10.1177/0194599820909438

2. Gürkov R, Pyykö I, Zou J, Kentala E. What is Menière's disease? A contemporary re-evaluation of endolymphatic hydrops. J Neurol. (2016) 263(Suppl. 1):S71-81. doi: 10.1007/s00415-015-7930-1

3. Merchant SN, Adams JC, Nadol JB Jr. Pathophysiology of Meniere's syndrome: are symptoms caused by endolymphatic hydrops? Otol Neurotol. (2005) 26:74-81. doi: 10.1097/00129492-200501000-00013

4. Committee on Hearing and Equilibrium guidelines for the diagnosis and evaluation of therapy in Menière's disease. American Academy of Otolaryngology-Head and Neck Foundation, Inc. Otolaryngol Head Neck Surg. (1995) 113:181-5. doi: 10.1016/S0194-5998(95)70102-8

5. Lopez-Escamez JA, Carey J, Chung WH, Goebel JA, Magnusson M, Mandala M, et al. Diagnostic criteria for Meniere's disease. J Vestib Res. (2015) 25:17. doi: 10.3233/VES-150549

6. Sobhy OA, Elmoazen DM, Abd-Elbaky FA. Towards a new staging of Ménière's disease: a vestibular approach. Acta Otorhinolaryngol Ital. (2019) 39:419-28. doi: 10.14639/0392-100X-2461

7. Reinshagen KL, Curtin HD. Radiological assessment of the vestibular system. Oper Tech Otolaryngol. (2019) 30:171-9. doi: 10.1016/j.otot.2019.07.011

8. Naganawa S, Nakashima T. Visualization of endolymphatic hydrops with MR imaging in patients with Ménière's disease and related pathologies: current status of its methods and clinical significance. Jpn J Radiol. (2014) 32:191204. doi: 10.1007/s11604-014-0290-4

9. Naganawa S, Sugiura M, Kawamura M, Fukatsu H, Sone M, Nakashima T. Imaging of endolymphatic and perilymphatic fluid at 3T after intratympanic administration of gadolinium-diethylene-triamine pentaacetic acid. AJNR Am J Neuroradiol. (2008) 29:724-6. doi: 10.3174/ajnr.A0894

10. Fiorino F, Pizzini FB, Beltramello A, Mattellini B, Barbieri F. Reliability of magnetic resonance imaging performed after intratympanic administration of gadolinium in the identification of endolymphatic hydrops in patients with Ménière's disease. Otol Neurotol. (2011) 32:472-7. doi: 10.1097/MAO.0b013e31820e7614

11. Attyé A, Eliezer M, Boudiaf N, Tropres I, Chechin D, Schmerber S, et al. MRI of endolymphatic hydrops in patients with Meniere's disease: a case-controlled study with a simplified classification based on saccular morphology. Eur Radiol. (2017) 27:3138-46. doi: 10.1007/s00330-0164701-z

12. Conte G, Caschera L, Calloni S, Barozzi S, Di Berardino F, Zanetti D, et al. MR imaging in Menière disease: is the contact between the vestibular endolymphatic space and the oval window a reliable biomarker? AJNR Am J Neuroradiol. (2018) 39:2114-9. doi: 10.3174/ajnr.A5841

13. Suga K, Kato M, Yoshida T, Nishio N, Nakada T, Sugiura S, et al. Changes in endolymphatic hydrops in patients with Ménière's disease treated conservatively for more than 1 year. Acta Otolaryngol. (2015) 135:86670. doi: 10.3109/00016489.2015.1015607

14. Claes G, Van den Hauwe L, Wuyts F, Van de Heyning P. Does intratympanic gadolinium injection predict efficacy of gentamicin

\section{FUNDING}

The costs of publication will be covered by Associazione Progetto Udire OdV, a charity supporting the research on deafness and vestibular disorders, listed in the Lombardy Region registry of non-profit organizations.

\section{SUPPLEMENTARY MATERIAL}

The Supplementary Material for this article can be found online at: https://www.frontiersin.org/articles/10.3389/fsurg. 2021.700271/full\#supplementary-material

partial chemolabyrinthectomy in Menière's disease patients? Eur Arch Otorhinolaryngol. (2012) 269:413-8. doi: 10.1007/s00405-011-1644-5

15. Ito $\mathrm{T}$, Inui $\mathrm{H}$, Miyasaka $\mathrm{T}$, Shiozaki $\mathrm{T}$, Matsuyama $\mathrm{S}$, Yamanaka $\mathrm{T}$, et al. Three-dimensional magnetic resonance imaging reveals the relationship between the control of vertigo and decreases in endolymphatic hydrops after endolymphatic sac drainage with steroids for Meniere's disease. Front Neurol. (2019) 10:46. doi: 10.3389/fneur.2019.00046

16. Moher D, Liberati A, Tetzlaff J, Altman DG, The PRISMA Group. Preferred reporting items for systematic reviews and meta-analyses: the PRISMA statement. PLoS Med. (2009) 6:e1000097. doi: 10.1371/journal.pmed.1000097

17. Gürkov R, Flatz W, Louza J, Strupp M, Ertl-Wagner B, Krause E. In vivo visualized endolymphatic hydrops and inner ear functions in patients with electrocochleographically confirmed Ménière’s disease. Otol Neurotol. (2012) 33:1040-5. doi: 10.1097/MAO.0b013e31825d9a95

18. Naganawa S, Yamazaki M, Kawai H, Bokura K, Sone M, Nakashima T. Imaging of Ménière's disease by subtraction of MR cisternography from positive perilymph image. Magn Reson Med Sci. (2012) 11:3039. doi: $10.2463 / \mathrm{mrms} .11 .303$

19. Sano R, Teranishi M, Yamazaki M, Isoda H, Naganawa S, Sone M, et al. Contrast enhancement of the inner ear in magnetic resonance images taken at 10 minutes or 4 hours after intravenous gadolinium injection. Acta Otolaryngol. (2012) 132:241-6. doi: 10.3109/00016489.2011.639085

20. Naganawa S, Yamazaki M, Kawai H, Bokura K, Sone M, Nakashima T. Imaging of Ménière's disease after intravenous administration of single-dose gadodiamide: utility of multiplication of MR cisternography and HYDROPS image. Magn Reson Med Sci. (2013) 12:63-8. doi: 10.2463/mrms.2012-0027

21. Seo YJ, Kim J, Choi JY, Lee WS. Visualization of endolymphatic hydrops and correlation with audio-vestibular functional testing in patients with definite Meniere's disease. Auris Nasus Larynx. (2013) 40:16772. doi: 10.1016/j.anl.2012.07.009

22. Naganawa S, Yamazaki M, Kawai H, Bokura K, Iida T, Sone M, et al. MR imaging of Ménière's disease after combined intratympanic and intravenous injection of gadolinium using HYDROPS2. Magn Reson Med Sci. (2014) 13:133-7. doi: 10.2463/mrms.2013-0061

23. Baráth K, Schuknecht B, Naldi AM, Schrepfer T, Bockisch CJ, Hegemann SC. Detection and grading of endolymphatic hydrops in Menière disease using MR imaging. AJNR Am J Neuroradiol. (2014) 35:1387-92. doi: 10.3174/ajnr.A3856

24. Liu Y, Jia H, Shi J, Zheng H, Li Y, Yang J, et al. Endolymphatic hydrops detected by 3-dimensional fluid-attenuated inversion recovery MRI following intratympanic injection of gadolinium in the asymptomatic contralateral ears of patients with unilateral Ménière's disease. Med Sci Monit. (2015) 21:701-7. doi: 10.12659/MSM.892383

25. Sepahdari AR, Ishiyama G, Vorasubin N, Peng KA, Linetsky M, Ishiyama A. Delayed intravenous contrast-enhanced 3D FLAIR MRI in Meniere's disease: correlation of quantitative measures of endolymphatic hydrops with hearing. Clin Imaging. (2015) 39:26-31. doi: 10.1016/j.clinimag.2014.09.014

26. Hornibrook J, Flook E, Greig S, Babbage M, Goh T, Coates M, et al. MRI inner ear imaging and tone burst electrocochleography in the diagnosis of Ménière's disease. Otol Neurotol. (2015) 36:110914. doi: 10.1097/MAO.0000000000000782 
27. Bykowski J, Harris JP, Miller M, Du J, Mafee MF. Intratympanic contrast in the evaluation of Menière disease: understanding the limits. AJNR Am J Neuroradiol. (2015) 36:1326-32. doi: 10.3174/ajnr.A4277

28. Attyé A, Dumas G, Troprès I, Roustit M, Karkas A, Banciu E, et al. Recurrent peripheral vestibulopathy: is MRI useful for the diagnosis of endolymphatic hydrops in clinical practice? Eur Radiol. (2015) 25:30439. doi: 10.1007/s00330-015-3712-5

29. Wu Q, Dai C, Zhao M, Sha Y. The correlation between symptoms of definite Meniere's disease and endolymphatic hydrops visualized by magnetic resonance imaging. Laryngoscope. (2016) 126:974-9. doi: 10.1002/lary.25576

30. Pakdaman MN, Ishiyama G, Ishiyama A, Peng KA, Kim HJ, Pope $\mathrm{WB}$, et al. Blood-labyrinth barrier permeability in Menière disease and idiopathic sudden sensorineural hearing loss: findings on delayed postcontrast 3D-FLAIR MRI. AJNR Am J Neuroradiol. (2016) 37:19038. doi: 10.3174/ajnr.A4822

31. Keller JH, Hirsch BE, Marovich RS, Branstetter BF 4th. Detection of endolymphatic hydrops using traditional MR imaging sequences. Am J Otolaryngol. (2017) 38:442-6. doi: 10.1016/j.amjoto.2017.01.038

32. Choi JE, Kim YK, Cho YS, Lee K, Park HW, Yoon SH, et al. Morphological correlation between caloric tests and vestibular hydrops in Ménière's disease using intravenous Gd enhanced inner ear MRI. PLoS ONE. (2017) 12:e0188301. doi: 10.1371/journal.pone.0188301

33. Okumura $\mathrm{T}$, Imai $\mathrm{T}$, Takimoto $\mathrm{Y}$, Takeda $\mathrm{N}$, Kitahara $\mathrm{T}$, Uno $\mathrm{A}$, et al. Assessment of endolymphatic hydrops and otolith function in patients with Ménière's disease. Eur Arch Otorhinolaryngol. (2017) 274:141321. doi: 10.1007/s00405-016-4418-2

34. Yoshida T, Sugimoto S, Teranishi M, Otake H, Yamazaki M, Naganawa S, et al. Imaging of the endolymphatic space in patients with Ménière's disease. Auris Nasus Larynx. (2018) 45:33-8. doi: 10.1016/j.anl.2017.02.002

35. Wesseler A, Óvári A, Javorkova A, Kwiatkowski A, Meyer JE, Kivelitz DE. Diagnostic value of the magnetic resonance imaging with intratympanic gadolinium administration (IT-Gd MRI) versus audio-vestibular tests in Menière's disease: IT-Gd MRI makes the difference. Otol Neurotol. (2019) 40:e225-32. doi: 10.1097/MAO.0000000000002082

36. Bier G, Bongers MN, Schabel C, Heindel W, Ernemann U, Hempel JM. In vivo assessment of an endolymphatic hydrops gradient along the cochlea in patients with Menière's disease by magnetic resonance imaging-a pilot study. Otol Neurotol. (2018) 39:e1091-9. doi: 10.1097/MAO.0000000000002016

37. Quatre R, Attyé A, Karkas A, Job A, Dumas G, Schmerber S. Relationship between audio-vestibular functional tests and inner ear MRI in Meniere's disease. Ear Hear. (2019) 40:168-76. doi: 10.1097/AUD.0000000000000584

38. Pérez-Fernández N, Dominguez P, Manrique-Huarte R, Calavia D, Arbizu L, Garcia-Eulate R, et al. Endolymphatic hydrops severity in magnetic resonance imaging evidences disparate vestibular test results. Auris Nasus Larynx. (2019) 46:210-7. doi: 10.1016/j.anl.2018.08.014

39. Bernaerts A, Vanspauwen R, Blaivie C, van Dinther J, Zarowski A, Wuyts FL, et al. The value of four stage vestibular hydrops grading and asymmetric perilymphatic enhancement in the diagnosis of Menière's disease on MRI. Neuroradiology. (2019) 61:421-9. doi: 10.1007/s00234-019-02155-7

40. Eliezer M, Maquet C, Horion J, Gillibert A, Toupet M, Bolognini B, et al. Detection of intralabyrinthine abnormalities using post-contrast delayed 3DFLAIR MRI sequences in patients with acute vestibular syndrome. Eur Radiol. (2019) 29:2760-9. doi: 10.1007/s00330-018-5825-0

41. Guo P, Sun W, Shi S, Zhang F, Wang J, Wang W. Quantitative evaluation of endolymphatic hydrops with MRI through intravenous gadolinium administration and VEMP in unilateral definite Meniere's disease. Eur Arch Otorhinolaryngol. (2019) 276:993-1000. doi: 10.1007/s00405-018-05267-7

42. Ohashi T, Naganawa S, Takeuchi A, Katagiri T, Kuno K. Quantification of endolymphatic space volume after intravenous administration of a single dose of gadolinium-based contrast agent: 3D-real inversion recovery versus HYDROPS-Mi2. Magn Reson Med Sci. (2020) 19:11924. doi: 10.2463/mrms.mp.2019-0013

43. Gerb J, Ahmadi SA, Kierig E, Ertl-Wagner B, Dieterich M, Kirsch V. VOLT: a novel open-source pipeline for automatic segmentation of endolymphatic space in inner ear MRI. J Neurol. (2020) 267(Suppl 1):18596. doi: 10.1007/s00415-020-10062-8

44. Nahmani S, Vaussy A, Hautefort C, Guichard JP, Guillonet A, Houdart E, et al. Comparison of enhancement of the vestibular perilymph between variable and constant flip angle-delayed 3D-FLAIR sequences in Menière disease. AJNR Am J Neuroradiol. (2020) 41:706-11. doi: 10.3174/ajnr.A6483

45. Cho YS, Cho K, Park CJ, Chung MJ, Kim JH, Kim K, et al. Automated measurement of hydrops ratio from MRI in patients with Ménière's disease using CNN-based segmentation. Sci Rep. (2020) 10:7003. doi: 10.1038/s41598-020-63887-8

46. van Steekelenburg JM, van Weijnen A, de Pont LMH, Vijlbrief OD, Bommeljé CC, Koopman JP, et al. Value of endolymphatic hydrops and perilymph signal intensity in suspected Ménière disease. AJNR Am J Neuroradiol. (2020) 41:529-34. doi: 10.3174/ajnr.A6410

47. Pai I, Mendis S, Murdin L, Touska P, Connor S. Magnetic resonance imaging of Ménière's disease: early clinical experience in a UK centre. J Laryngol Otol. (2020) 134:302-10. doi: 10.1017/S0022215120000626

48. Fukushima M, Ueno Y, Kitayama I, Akahani S, Inohara H, Takeda N. Assessment of the progression of vertical semicircular canal dysfunction and increased vestibular endolymphatic hydrops in patients with earlystage Ménière disease. JAMA Otolaryngol Head Neck Surg. (2020) 146:789800. doi: 10.1001/jamaoto.2020.1496

49. Kahn L, Hautefort C, Guichard JP, Toupet M, Jourdaine C, Vitaux $\mathrm{H}$, et al. Relationship between video head impulse test, ocular and cervical vestibular evoked myogenic potentials, and compartmental magnetic resonance imaging classification in menière's disease. Laryngoscope. (2020) 130:E444-52. doi: 10.1002/lary.28362

50. Gürkov R, Lutsenko V, Babkina T, Valchyshyn S, Situkho M. Clinical high-resolution imaging and grading of endolymphatic hydrops in hydropic ear disease at $1.5 \mathrm{~T}$ using the two-slice grading for vestibular endolymphatic hydrops in less than $10 \mathrm{~min}$. Eur Arch Otorhinolaryngol. (2021). doi: 10.1007/s00405-021-06731-7. [Epub ahead of print].

51. He B, Zhang F, Zheng H, Sun X, Chen J, Chen J, et al. The correlation of a 2D volume-referencing endolymphatic-hydrops grading system with extratympanic electrocochleography in patients with definite ménière's disease. Front Neurol. (2021) 11:595038. doi: 10.3389/fneur.2020.595038

52. Zhang W, Hui L, Zhang B, Ren L, Zhu J, Wang F, et al. The correlation between endolymphatic hydrops and clinical features of Meniere disease. Laryngoscope. (2021) 131:E144-50. doi: 10.1002/lary.28576

53. Sluydts M, Bernaerts A, Casselman JW, De Foer B, Blaivie C, Zarowski A, et al. The relationship between cochleovestibular function tests and endolymphatic hydrops grading on MRI in patients with Menière's disease. Eur Arch Otorhinolaryngol. (2021). doi: 10.1007/s00405-021-06610-1. [Epub ahead of print].

54. Carfrae MJ, Holtzman A, Eames F, Parnes SM, Lupinetti A. 3 Tesla delayed contrast magnetic resonance imaging evaluation of Ménière's disease. Laryngoscope. (2008) 118:501-5. doi: 10.1097/MLG.0b013e31815c1a61

55. Shi S, Zhou F, Wang W. 3D-real IR MRI of Meniere's disease with partial endolymphatic hydrops. Am J Otolaryngol. (2019) 40:589-93. doi: 10.1016/j.amjoto.2019.05.015

56. Iida $T$, Teranishi $M$, Yoshida $T$, Otake $H$, Sone $M$, Kato $M$, et al. Magnetic resonance imaging of the inner ear after both intratympanic and intravenous gadolinium injections. Acta Otolaryngol. (2013) 133:4348. doi: 10.3109/00016489.2012.753640

57. Gürkov R. Menière and friends: imaging and classification of hydropic ear disease. Otol Neurotol. (2017) 38:e53944. doi: 10.1097/MAO.0000000000001479

58. Tagaya M, Yamazaki M, Teranishi M, Naganawa S, Yoshida T, Otake H, et al. Endolymphatic hydrops and blood-labyrinth barrier in Ménière's disease. Acta Otolaryngol. (2011) 131:474-9. doi: 10.3109/00016489.2010.5 34114

59. Nakashima T, Naganawa S, Sugiura M, Teranishi M, Sone M, Hayashi H, et al. Visualization of endolymphatic hydrops in patients with meniere's disease. Laryngoscope. (2007) 117:415-20. doi: 10.1097/MLG.0b013e31802c300c

60. Nakashima T, Naganawa S, Pyykko I, Gibson WP, Sone M, Nakata S, et al. Grading of endolymphatic hydrops using magnetic resonance imaging. Acta Otolaryngol Suppl. (2009) 560:5-8. doi: 10.1080/00016480902729827

61. Gluth MB. On the relationship between Menière's disease and endolymphatic hydrops. Otol Neurotol. (2020) 41:2429. doi: 10.1097/MAO.0000000000002502

62. Van der Lubbe MFJA, Vaidyanathan A, Van Rompaey V, Postma AA, Bruintjes TD, Kimenai DM, et al. The "hype" of hydrops in classifying 
vestibular disorders: a narrative review. J Neurol. (2020) 267(Suppl. 1):197211. doi: $10.1007 / \mathrm{s} 00415-020-10278-8$

63. Morita Y, Takahashi K, Ohshima S, Yagi C, Kitazawa M, Yamagishi T, et al. Is vestibular Meniere's disease associated with endolymphatic hydrops? Front Surg. (2020) 7:601692. doi: 10.3389/fsurg.2020.601692

64. Eliezer M, Poillon G, Horion J, Lelion P, Gerardin E, Magne N, et al. MRI diagnosis of saccular hydrops: comparison of heavily-T2 FIESTA-C and 3D-FLAIR sequences with delayed acquisition. J Neuroradiol. (2019). doi: 10.1016/j.neurad.2019.04.005. [Epub ahead of print].

65. Zou J, Wang Z, Chen Y, Zhang G, Chen L, Lu J. MRI detection of endolymphatic hydrops in Meniere's disease in 8 minutes using MIIRMR and a 20-channel coil after targeted gadolinium delivery. World $J$ Otorhinolaryngol Head Neck Surg. (2020) 5:180-7. doi: 10.1016/j.wjorl.2019. 04.001

66. Vaidyanathan A, van der Lubbe MFJA, Leijenaar RTH, van Hoof M, Zerka F, Miraglio B, et al. Deep learning for the fully automated segmentation of the inner ear on MRI. Sci Rep. (2021) 11:2885. doi: 10.1038/s41598-021-8 2289-y

67. Lee J, Kim ES, Lee Y, Lee K, Yoon DY, Ju YS, et al. Quantitative analysis of cochlear signal intensity on three-dimensional and contrast-enhanced fluid-attenuated inversion recovery images in patients with Meniere's disease: correlation with the pure tone audiometry test. J Neuroradiol. (2019) 46:307-11. doi: 10.1016/j.neurad.2019.03.010

Conflict of Interest: The authors declare that the research was conducted in the absence of any commercial or financial relationships that could be construed as a potential conflict of interest.

Publisher's Note: All claims expressed in this article are solely those of the authors and do not necessarily represent those of their affiliated organizations, or those of the publisher, the editors and the reviewers. Any product that may be evaluated in this article, or claim that may be made by its manufacturer, is not guaranteed or endorsed by the publisher.

Copyright (c) 2021 Zanetti, Conte, Scola, Casale, Lilli and Di Berardino. This is an open-access article distributed under the terms of the Creative Commons Attribution License (CC BY). The use, distribution or reproduction in other forums is permitted, provided the original author(s) and the copyright owner(s) are credited and that the original publication in this journal is cited, in accordance with accepted academic practice. No use, distribution or reproduction is permitted which does not comply with these terms. 